Copyright @ The Author(s), 2021. Published by Cambridge University Press on behalf of Canadian Neurological Sciences Federation.

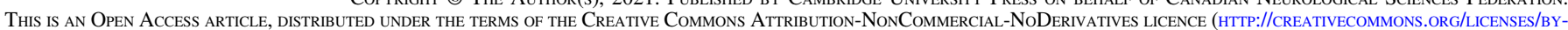

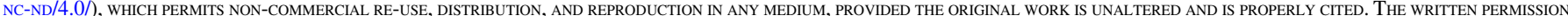
of CAmbridge University Press must be OBTAINed fOR COMMercial RE-USE OR IN ORDER to CREATE a DERIVATIVE WORK.

\title{
Guillain-Barre Syndrome with COVID-19
}

\author{
Tychicus Chen (D), Hannah R. Briemberg
}

Keywords: Guillain barre, Infectious diseases, Magnetic resonance imaging, Epidemiology

doi:10.1017/cjn.2021.108

Can J Neurol Sci. 2022; 49: 414-415

A 65-year-old woman presented to the hospital with low back pain, leg weakness, and paresthesias over 1 day. 15 days prior, she tested positive for coronavirus disease-2019 (COVID-19) on nasopharyngeal swab, after 2 days of cough. On initial examination, she was afebrile with normal vital signs. Cranial nerves and upper extremities were unremarkable with no weakness. Lower extremity strength was Medical Research Council grade 4/5 throughout. Deep tendon reflexes were present except for the knees. Sensation was reduced to pain and temperature in the hands and lateral lower legs bilaterally, and vibration in the distal fingers and toes. The next day she was intubated after an aspiration event, although did not require supplemental oxygen previously and chest radiography did not show any opacity throughout hospitalization. Repeat examination revealed quadriparesis, bifacial weakness, and diffuse areflexia. Cerebrospinal fluid (CSF) demonstrated normal glucose with markedly elevated protein $(2.50 \mathrm{~g} / \mathrm{L}$, normal $0.15-0.45 \mathrm{~g} / \mathrm{L})$ and mild pleocytosis $\left(9 \times 10^{6} / \mathrm{L}\right.$, normal $\left.<5 \times 10^{6} / \mathrm{L}\right)$ characteristic of albuminocytologic dissociation. Initial nasopharyngeal swab remained positive for COVID-19, followed by two consecutive negative tests 14 days later. CSF PCR was also negative. Magnetic resonance imaging demonstrated enhancement of cranial nerves (Figure 1) and caudal nerve roots (Figure 2). Nerve conduction studies performed 4 weeks after symptom onset revealed diffusely reduced motor amplitudes, prolonged distal latencies, conduction block, and temporal dispersion in ulnar, fibular, and tibial motor nerves, with absent F-waves in the lower extremities, prolonged in the upper extremities. Radial and sural sensory responses were preserved.

Nerve root enhancement of the cauda equina is seen frequently in Guillain-Barre syndrome (GBS). ${ }^{1,2}$ Of 1200 patients admitted to the hospital with COVID-19 in Northern Italy over a 1 month period, 5 were diagnosed with GBS, with MRI showing enhancement of bilateral facial nerves in 1 patient and caudal nerve roots in $2{ }^{3}$ Miller Fisher syndrome and polycranialis continua - variants of GBS with cranial nerve involvement - have also been described in association with COVID-19, ${ }^{4}$ as has facial diplegia. ${ }^{5}$ In a separate review of 37 published cases of GBS associated with COVID-19, neuroimaging was reported in less than half: facial nerve enhancement was seen in 2 patients and lumbosacral nerve root enhancement or radiculitis were seen in 4 others. ${ }^{6}$ Weakness

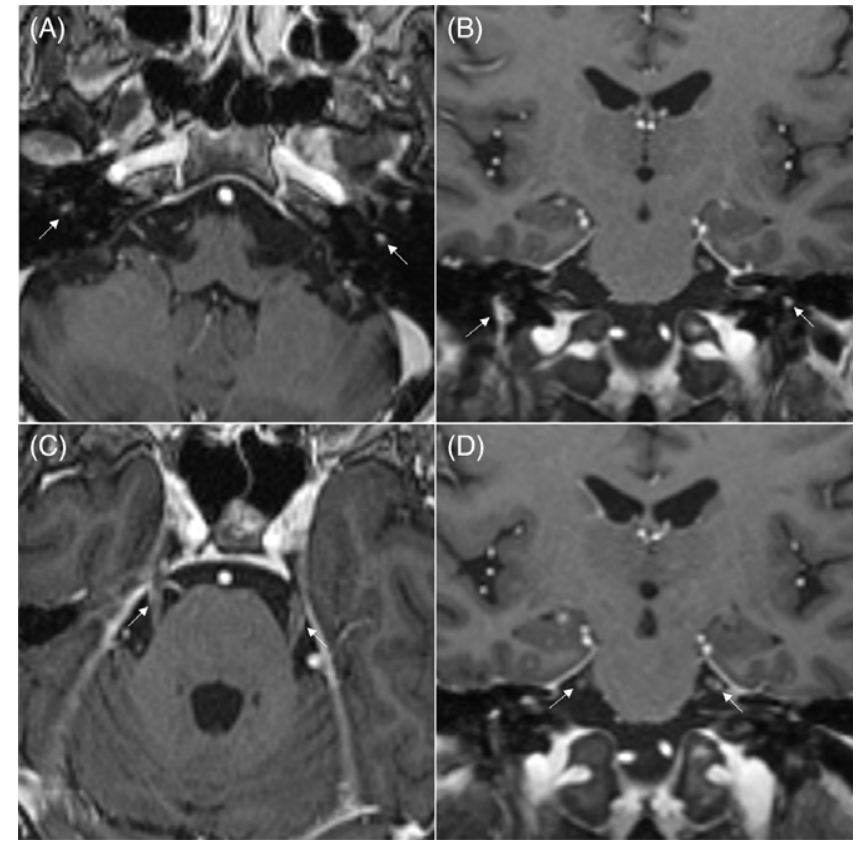

Figure 1: Axial and coronal T1 post-gadolinium MRI brain showing abnormal enhancement (arrows) of the bilateral intracanalicular facial nerves $(A, B)$ and cisternal trigeminal nerves $(C, D)$.

began an average of 11 days (range 3-28 days) post-COVID-19 symptom onset. ${ }^{6}$ Albuminocytologic dissociation was observed in three quarters. ${ }^{6} 33$ patients were treated with intravenous immunoglobulin, and 3 with plasmapheresis, with response to therapy reported in $89 \% .^{6}$

Evidence of an association between GBS and COVID-19 is conflicting. ${ }^{7,8}$ In the United Kingdom, the incidence of GBS fell between March and May 2020 when compared to the same months in 2016-2019. ${ }^{7}$ In Northern Italy, the incidence was found to be 2.6 times higher in March and April 2020 compared with the same period in $2019 .{ }^{8}$ It appears that GBS can rarely be associated with COVID-19. Whether this represents pure coincidence or is related to individual-specific predisposing factors remains to be determined. Several studies of CSF in COVID-19 patients with neurological symptoms report negative PCR testing,

From the Division of Neurology, Department of Medicine, University of British Columbia, Vancouver, British Columbia, Canada (TC, HRB)

Received April 14, 2021. Final Revisions Submitted May 2, 2021. Date of Acceptance May 5, 2021.

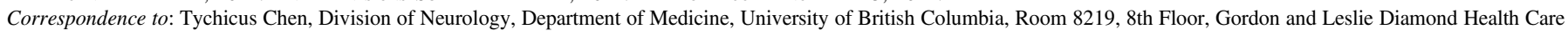
Centre, 2775 Laurel Street, Vancouver, BC V5Z 1M9, Canada. Email: tychicus@mail.ubc.ca 


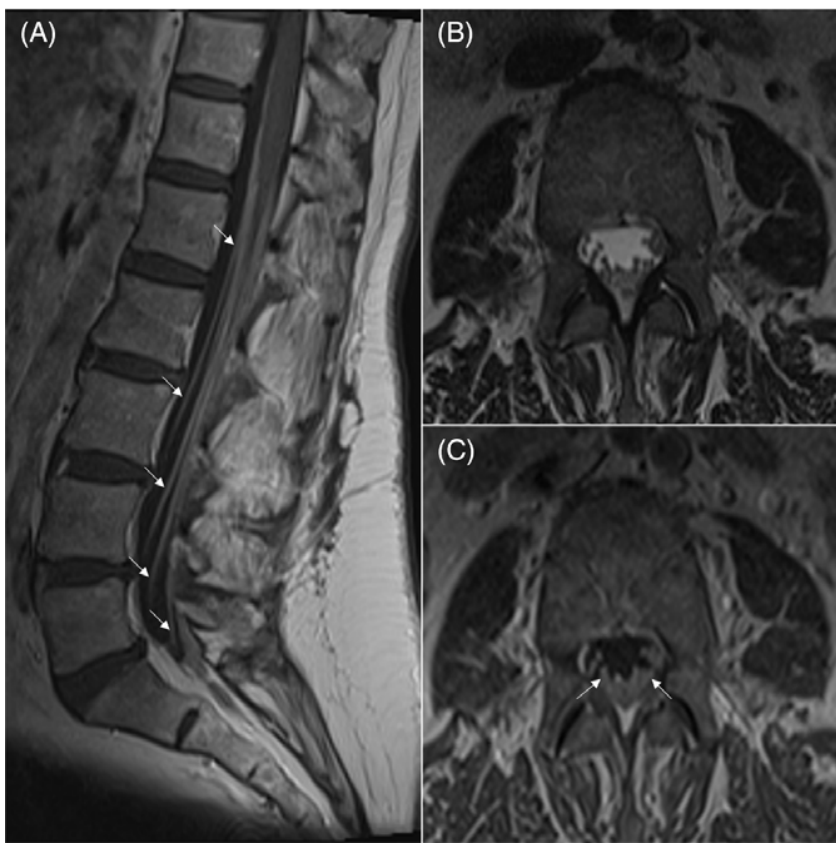

Figure 2: Sagittal T1 post-gadolinium MRI lumbar spine (A) showing smooth enhancement (arrows) of the caudal nerve roots. Axial $T 2$-weighted $(B)$ and $T 1$ post-gadolinium $(C)$ views again demonstrating nerve root enhancement throughout the cauda equina (arrows).

suggesting an indirect mechanism ${ }^{9}$ : one study of 30 patients included 2 with GBS and 1 with Miller Fisher syndrome, ${ }^{10}$ and another of 31 patients included 4 with polyneuropathy. ${ }^{11}$ This case highlights the MRI findings of facial nerve, trigeminal nerve, and lumbosacral nerve root enhancement in a patient with GBS, which may occur in association with COVID-19.

\section{ACKNOWLedgments}

The authors wish to thank the patient for permission to publish this case report.

\section{Disclosures}

The authors report no disclosures relevant to this manuscript.

\section{Statement of Authorship}

Both authors contributed equally to this manuscript. Authors take full responsibility for the data collected, analysis, interpretation, and conduct of this report.

\section{REFERENCES}

1. Leonhard SE, Mandarakas MR, Gondim FAA, et al. Diagnosis and management of Guillain-Barré syndrome in ten steps. Nat Rev Neurol. 2019;15:671-83.

2. Gorson KC, Ropper AH, Muriello MA, Blair R. Prospective evaluation of MRI lumbosacral nerve root enhancement in acute Guillain-Barré syndrome. Neurology. 1996;47:813-7.

3. Toscano G, Palmerini F, Ravaglia S, et al. Guillain-Barré syndrome associated with SARS-CoV-2. N Engl J Med. 2020;382: 2574-6.

4. Gutiérrez-Ortiz C, Méndez-Guerrero A, Rodrigo-Rey S, et al. Miller Fisher syndrome and polyneuritis cranialis in COVID-19. Neurology. 2020;95:e601-5.

5. Juliao Caamaño DS, Alonso Beato R. Facial diplegia, a possible atypical variant of Guillain-Barré syndrome as a rare neurological complication of SARS-CoV-2. J Clin Neurosci. 2020;77:230-2.

6. Caress JB, Castoro RJ, Simmons Z, et al. COVID-19-associated Guillain-Barré syndrome: the early pandemic experience. Muscle Nerve. 2020;62:485-91.

7. Keddie S, Pakpoor J, Mousele C, et al. Epidemiological and cohort study finds no association between COVID-19 and Guillain-Barré syndrome. Brain. 2021;144:682-93.

8. Filosto M, Cotti Piccinelli S, Gazzina S, et al. Guillain-Barré syndrome and COVID-19: an observational multicentre study from two Italian hotspot regions. J Neurol Neurosurg Psychiatry. 2020:1-6.

9. Lucchese G. Cerebrospinal fluid findings in COVID-19 indicate autoimmunity. Lancet Microbe. 2020;1:e242.

10. Neumann B, Schmidbauer ML, Dimitriadis K, et al. Cerebrospinal fluid findings in COVID-19 patients with neurological symptoms. J Neurol Sci. 2020;418:117090.

11. Bellon M, Schweblin C, Lambeng N, et al. Cerebrospinal fluid features in SARS-CoV-2 RT-PCR positive patients. Clin Infect Dis. 2020:ciaa1165. 